LA GRANJA:

REVISTA DE

CIENCIAS DE LA VIDA

pISSN:1390-3799; eISSN:1390-8596

http:/ / doi.org/10.17163/lgr.n28.2018.04
Artículo cientifico / Scientific paper

HidROLOGÍA

\title{
COMPONENTES DEL BALANCE HÍDRICO EN LOS PÁRAMOS DE JATUNSACHA, ECUADOR
}

\author{
WATER BALANCE COMPONENTS IN THE PARAMO OF JATUNSACHA, ECUADOR \\ Sergio Fernando Torres Romero ${ }^{1,2 *}$ y Carlos Oswaldo Proaño Santos 3,4
}

\footnotetext{
${ }^{1}$ Universidad Nacional de Loja, Avenida Pio Jaramillo Alvarado, Loja, Ecuador.

${ }^{2}$ Universidad Nacional de Colombia, Bogotá, Colombia.

${ }_{3}^{3}$ Escuela Politécnica Nacional, Ladron de Guevara E11-253, Quito, Ecuador.

${ }^{4}$ IHE-UNESCO, Delft, Holanda.
}

*Autor para correspondencia: seftorresro@unal.edu.co

\section{Resumen}

El páramo es una zona biogeográfica de alta montaña que comúnmente se extiende entre el límite superior del bosque andino hasta por debajo de las nieves perpetuas. Hidrológicamente este ecosistema presenta una buena regulación de los caudales bases, como resultado de la interacción de los componentes precipitación, humedad del suelo y evapotranspiración, lo que permite el abastecimiento continuo del recurso hídrico para las poblaciones ubicadas en las cuencas medias y bajas de la región Andina. La presente investigación tiene como objetivo evaluar el comportamiento de los principales parámetros que caracterizan el balance hídrico en los páramos de Jatunsacha, con base en la recolección, análisis y procesamiento de la información hidroclimatologica de estaciones ubicadas en los páramos de Antisana, como mecanismo de apoyo en la toma de decisiones para el manejo y conservación de zonas de recarga hídrica de la parte norte del Ecuador. De acuerdo con los resultados, la dinámica del régimen hidrológico en la zona de estudio está determina por eventos de lluvia de baja intensidad, volumen y duración pero muy frecuentes, por un contenido de humedad del suelo entre capacidad de campo y punto de saturación, por una evapotranspiración relativamente baja, por un caudal muy variable que genera un coeficiente de escorrentía bajo y por una percolación alta típico en zonas con geología porosa.

Palabras claves: Precipitación, Caudal, Evapotranspiración real, dinámica de la humedad del suelo, hidrología. 


\begin{abstract}
The neotropical paramo is a high mountain biome distributed from the upper side of the andean forest to the permanent snow line of such ecosystems. From a hydrological point of view, this kind of ecosystem is characterized by maintaining a constant amount of base flow, as a result of the interaction of several of its water balance components such as: precipitation; soil moisture and evapotranspiration. Therefore, Andean communities and population located downstream of the catchment are able to count with enough water most of the year. The main objective of this research work is to perform an assessment of the influence of such water balance in the zone of Jatunsacha paramos. This research work is carried out based on the collection, evaluation and processing of hydrologic data from stages located in the zone of the Antisana. Such information will be used as a decision making support tool for the conservation and management of recharge zones in the northern part of Ecuador. In accordance with the obtained results, the dynamics of the hydrologic regime depends on: the frequent occurrence of low intensity, low volume and short duration rainfall events; soil moisture variability at both field capacity and the point of saturation; a relatively low evapotranspiration; a highly variable flow discharge that generates a low discharge coefficient; and, a high rate of percolation which is typical from porous zones such as the volcanic paramo zones.
\end{abstract}

Keywords: Precipitation, discharge, evapotranspiration, soil moisture dynamics, hydrology.

Forma sugerida de citar: Torres Romero, S. F. y Proaño Santos, C. O. 2018. Componentes del balance hídrico en los páramos de jatunsacha, Ecuador. La Granja: Revista de Ciencias de la Vida. Vol. 28(2):52-66. http://doi.org/10.17163/lgr.n28.2018.04. 


\section{Introducción}

Los páramos son ecosistemas naturales distribuidos en toda la zona tropical, específicamente a los $8^{\circ}$ latitud Norte y $11^{\circ}$ latitud Sur (Hofstede et al., 2003). En la parte Andina forman un corredor entre la cordillera de Mérida, en Venezuela, hasta la depreciación de Huancabamba al norte del Perú; pero también existen complejos más separados como los páramos en Costa Rica y la Sierra Nevada de Santa Marta (Hofstede et al., 2003; Buytaert, CuestaCamacho y Tobón, 2011; Luteyn et al., 1999).

Más de 100 millones de personas en los Andes y en las partes montañosas de África y Nueva Guinea dependen indirectamente del abastecimiento de líquido vital de este ecosistema (Hofstede et al., 2003), que se ve afectado por una degradación paulatina, causada por el cambio de uso del suelo e incremento de la demanda del recurso hídrico para el consumo, riego y generación de hidroelectricidad (Buytaert et al., 2005; Buytaert, Célleri, De Bièvre, Cisneros, Wyseure, Deckers y Hofstede, 2006; Buytaert, Iniguez y De Bievre, 2007; Crespo et al., 2011; Harden et al., 2013).

A pesar de la importancia mencionada por su constante suministro de agua, a nivel científico la información de bases de datos hidrológicos y climatológicos son escasos y los procedimientos de tratamiento de información (pre-procesamiento) son casi inexistentes, situación que genera que la interpretación de resultados sea errónea, por lo que es necesario estandarizar protocolos para extracción de datos, pre-procesamiento, mantenimiento de equipos con diferentes tipos de sensores y almacenamiento de bases que puedan ser accesibles para estudios de carácter científico y que permitan a los tomadores de decisiones un uso y manejo adecuado del ecosistema de páramo.

La investigaciones en el ecosistema de páramo sobre el funcionamiento hidrológico mencionan que la precipitación para la región Andina varía entre $700 \mathrm{~mm}$ a $3000 \mathrm{~mm}$, con una variabilidad a escala local influenciada por la topografía irregular, la altitud, pendiente y orientación (Buytaert, Iniguez y De Bievre, 2007; Buytaert, Célleri, De Bièvre, Cisneros, Wyseure, Deckers y Hofstede, 2006; Celleri et al., 2007). Por otro lado estudios a nivel de microcuenca en el sur del Ecuador determinaron variaciones de evapotranspiración entre 0.6 a $2.51 \mathrm{~mm}$ día $^{-1}$ (Buytaert, 2004; Célleri y Feyen, 2009; Hofstede, 1995; Buytaert, Célleri, De Bièvre, Cisneros, Wy- seure, Deckers y Hofstede, 2006; Favier et al., 2008). De igual forma, Ochoa-Tocachi et al. (2016) evaluaron el coeficiente de escorrentía en cuencas de páramo ubicadas en los volcanes Antisana y Pichincha, obteniendo valores entre 8 y $13 \%$ que difieren de los resultados en los estudios de Tobón y Arroyave (2007); Buytaert, Iniguez y De Bievre (2007); Crespo et al. (2011); Guzmán et al. (2015) con valores publicados entre $53 \%$ y $73 \%$ para los páramos en Colombia y sur del Ecuador.

Como una respuesta al análisis realizado y con la finalidad de generar conocimiento sobre la funcionalidad hidrológica se planteó el presente trabajo de investigación en la cuenca del río Jatunsacha, en los páramos de Antisana, como parte de una iniciativa nacional de monitoreo hidrometeorológico del Fondo para la Protección del Agua FONAG, con el objetivo de evaluar el comportamiento de los principales parámetros que caracterizan el balance hídrico en los páramos de Jatunsacha.

\section{Materiales y métodos}

\section{$2.1 \quad$ Área de estudio}

La cuenca en estudio se encuentra ubicada en el páramo del Antisana, perteneciente a la unidad hidrográfica del río Jatunhuaycu, fuente abastecedora de agua potable para la cuidad de Quito en Ecuador; geográficamente se localiza en los $0^{\circ} 28^{\prime} 15^{\prime \prime}$ latitud Sur y $78^{\circ} 14^{\prime} 34^{\prime \prime}$ longitud Oeste. Jatunsacha que drena sus aguas hacia la cuenca del río Amazonas cubre un área de $2,1 \mathrm{~km}^{2}$, con una pendiente promedio del $20 \%$ distribuida en una gradiente altitudinal que varía entre los $4036 \mathrm{msnm}$ y $4520 \mathrm{msnm}$ (Figura 1), con intervención relativamente baja por ganado vacuno y sin aporte por flujo del glacial Antisana (Alvarado, 2009; Torres, 2016).

La geología de la cuenca está conformada por unidades que contienen rocas metamórficas del Terreno Loja, emplazadas en rocas volcánicas de la formación Pisayambo (Barberi et al., 1988), cubierta locamente por una serie de depósitos superficiales que incluyen materiales piroplásticos, glaciales y coluviales (Coltorti y Ollier, 2000; Lavenu et al., 1992; Alvarado, 2009; Bourdon et al., 2002). Los suelos generalmente son de origen volcánico y pertenecen al orden de Andisoles, con presencia de limos de color negro(Torres, 2016; Alvarado, 2009; OchoaTocachi et al., 2016). 
La cobertura vegetal en Jatunsacha forma parte del superpáramo seco (Cleef, 1981; Sklenár y Balslev, 2005) dominado por las especies vegetales como: Festuca vaginalis, Plantago nubigena, Astragalus geminiflorus, Biden sandicola, Conyza cardaminifolia, Calamagrostis mollis, Cerastium imbricatum y Silene thysanodes (Sklenár y Balslev, 2005).

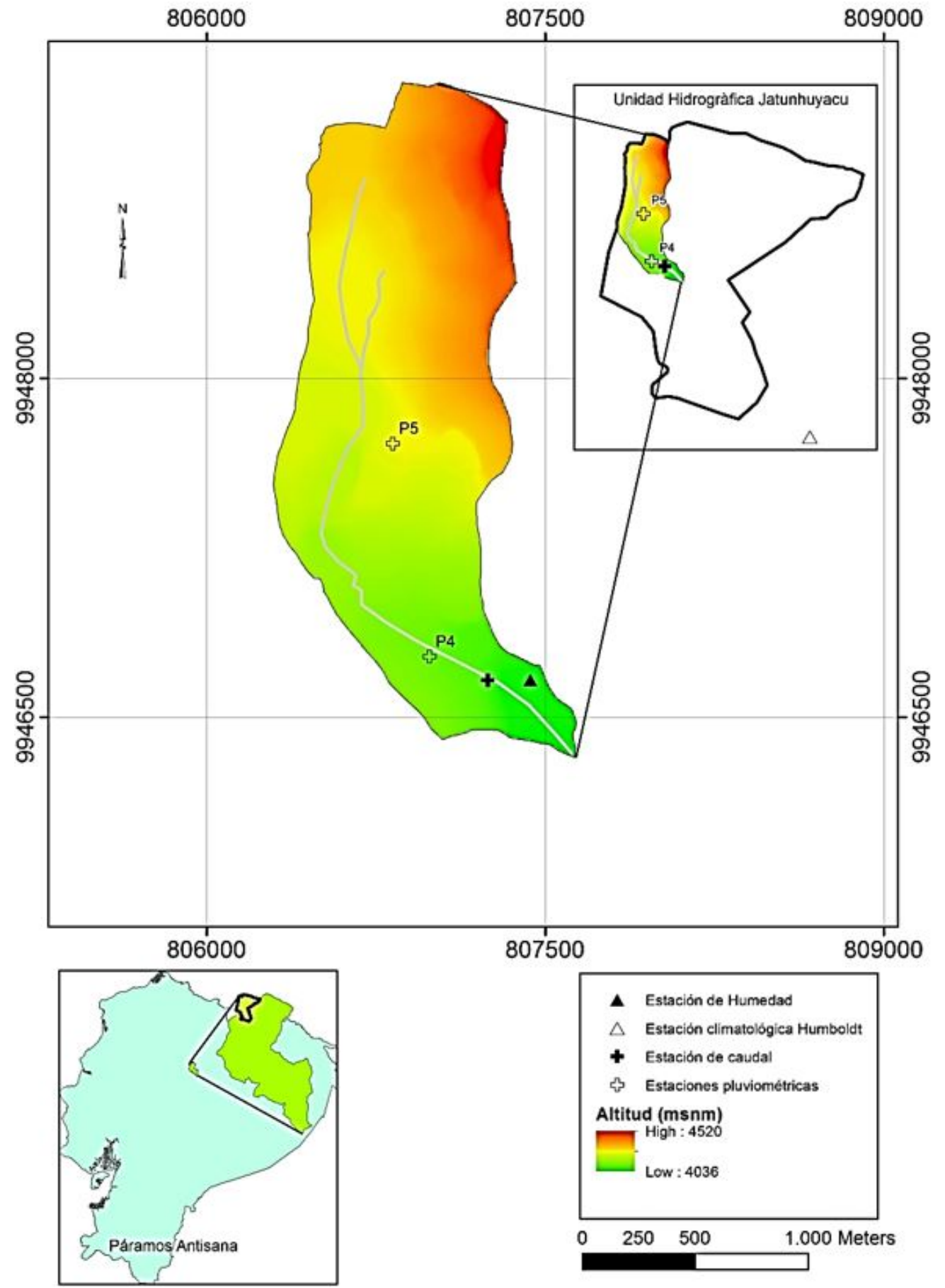

Figura 1. Ubicación geográfica de la cuenca y localización detallada de las estaciones pluviométricas, caudal, humedad del suelo y climatológica instaladas para el monitoreo hidrometeorológico. 


\subsection{Datos y métodos}

\subsubsection{Precipitación}

Para determinar las entradas por precipitación y su variabilidad temporal se instaló un pluviómetro marca TEXAS TE525MM, de cubeta electrónica basculante, con resolución de 0,1 $\mathrm{mm}$ asociados a un datalogger Campbell Scientific Cr-200X, colocados tanto en la parte baja y alta de la cuenca (Figura 1), que registraron información del periodo 1 de enero del 2014 al 30 de abril del 2015. En esta parte de la metodología se buscó analizar la lluvia a escala intra-anual para conocer cuánto llueve en el páramo y cómo se distribuye mensualmente.

Para la caracterización de los eventos de precipitación se empleó la información del pluviómetro cuatro (P4), que se localiza en la parte baja de la cuenca (Figura 1); esto por presentar una serie de registros más completa. Con la base de datos de lluvia agregada cada cinco minutos y con los resultados del ciclo anual de la precipitación (Figura 2) se caracterizaron los eventos de lluvia en cuatro períodos: período seco que comprende los meses de diciembre, enero, julio y agosto; período lluvioso marzo, abril, mayo y octubre; transición de seco a húmedo febrero y septiembre y transición de húmedo a seco junio y noviembre. Aunque no existe un criterio estandarizado para definir un evento de una serie de tiempo (Tokay et al., 2003), para la zona de estudio la separación se estableció en un tiempo máximo de una hora entre dos tips consecutivos; esto en referencia algunos estudios (Tokay et al., 2003; Holwerda et al., 2006; Nystuen, 1999) que fijaron los tiempos máximos entre 15 minutos y dos horas.

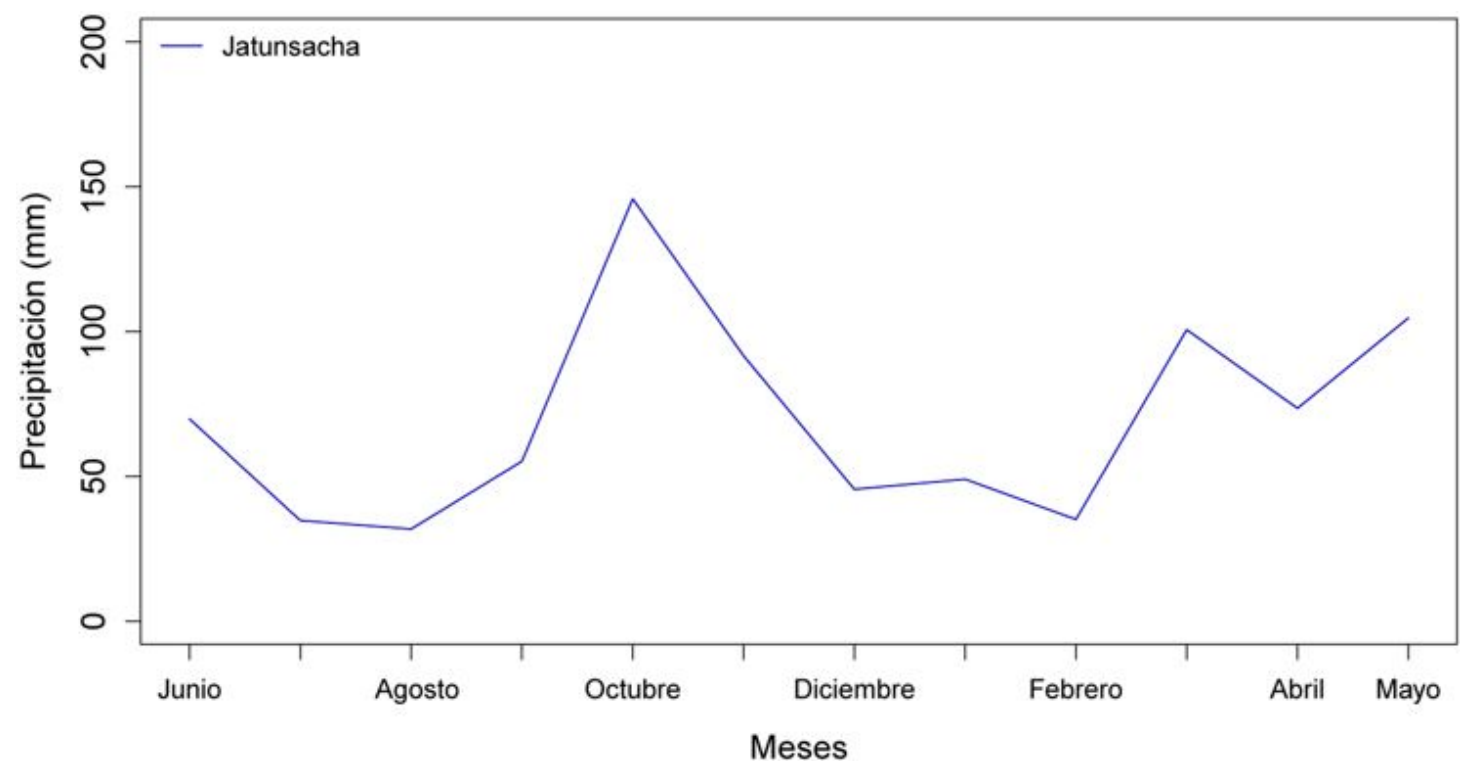

Figura 2. Estacionalidad del ciclo anual de la precipitación para la zona de estudio.

Con la base de datos en el programa $\mathrm{R}$ Studio se calculó la magnitud, duración, intensidad y número de eventos que es una medida directa de la frecuencia. La magnitud se estableció sumando los montos registrados en cada evento, la duración corresponden al tiempo transcurrido en cada evento, la intensidad se determinó dividiendo la magnitud entre el tiempo efectivo donde se registró la entrada por precipitación durante el evento; y la frecuencia se calculó sumando el número de eventos en cada período analizado. Para cada variable se realizó una caracterización de su función de distribución de probabilidad, que permite extraer límites de confianza al $95 \%$ para el acotamiento de su comportamiento intrínseco.

\subsubsection{Comportamiento de la humedad del suelo}

Para caracterizar el comportamiento de la humedad del suelo se instalaron instrumentos automáticos, basados en la mediciones de la permisividad dieléctrica del suelo, denominados TDR Time Domain 
Reflectometer (Jones, Wraith y Or, 2002), que son sensores bimetálicos colocados en forma horizontal en el horizonte superficial (A) a una profundidad de $10 \mathrm{~cm}$ y en el horizonte $2 \mathrm{Ab}$ a $70 \mathrm{~cm}$, dentro de una calicata excavada en una ladera de la cuenca (Figura 1), asociados a un dataloggers marca Campbell Scientific Cr-200X que registró información del contenido de agua del suelo, con una resolución de cinco minutos.

Como consecuencia de las singulares características de los suelos de cenizas volcánicas como son alta porosidad, baja densidad, presencia de vidrio volcánico y alta retención de humedad (Buytaert, 2004; Buytaert, Iniguez y De Bievre, 2007; Nanzyo, Shoji y Dahlgren, 1993); y en base a las recomendaciones de Blume, Zehe y Bronstert (2007) de calibrar las ecuaciones internas de los dataloggers se utilizó la ecuación propuesta por Guarderas (2015) para transformar las series de tiempo de tránsito registrado en el sensor, a valores de contenido volumétrico; relación determinada en base a un muestreo en campo en los dos horizontes.

Con las series de datos completas de humedad del suelo promedio agregados a escala diaria del periodo 30 de abril del 2014 al 13 de marzo del 2015, se graficó y analizó la curva dinámica de humedad para poder caracterizar el comportamiento del contenido de agua de los suelos en la cuenca Jatunsacha. Adicionalmente, se muestreó en campo por triplicado en cada horizonte la densidad aparente, con base en la relación masa de la muestra seca al horno durante 24 horas a $105{ }^{\circ} \mathrm{C}$ y el volumen de los cilindros (Grossman, 2002); materia orgánica a través del método de Walkey y Black (Black, 1965), porosidad total que se estableció a partir de la relación densidad aparente y densidad real (Klute, 1986a); conductividad hidráulica saturada donde se utilizó el método de carga hidráulica constante (Klute y Dirksen, 1996), potencial de hidrógeno $\mathrm{pH}$ a través de la relación 1:1 entre el agua y suelo y la curva de retención de humedad, considerando los puntos característicos: capacidad de campo, punto de saturación y punto de marchitez permanente, que se determinaron en el laboratorio de física de suelos de la Universidad Nacional de Colombia, sede Medellín, a partir de muestras sin disturbar tomadas con anillos de acero inoxidable de $5 \mathrm{~cm}$ de diámetro y $1 \mathrm{~cm}$ de altura, sometidas a saturación por capilaridad para posteriormente ser trasladadas a los platos con membranas; calibradas en diferentes presiones dentro de las "ollas de presión de Richard" y secadas en estufa a temperatura constante (Klute, 1986b).

\subsubsection{Evapotranspiración real}

Para el cálculo de la Evapotranspiración real (ETr) se utilizó la relación que integra el comportamiento climático, las características particulares de la vegetación y las limitaciones debido a la disponibilidad de agua a nivel de cuenca en el páramo del Antisana (Allen et al., 1998; Buytaert, Iñiguez, Celleri, De Biévre, Wyseure y Deckers, 2006; Guzmán et al., 2015), que se expresa de la siguiente manera (Ecuación 1):

$$
E T r=E T a \times K c \times K s
$$

En donde: ETr es la evapotranspiración real (mm día $\left.{ }^{-1}\right)$, ETa es la evapotranspiración de referencia (mm día ${ }^{-1}$ ), Kc es el coeficiente de cultivo (adimensional) y Ks el factor de estrés hídrico (adimensional).

Para la determinación de ETa se aplicó la ecuación de Penman-Monteith (Allen et al., 1998) (Ecuación 2), con datos procedentes de la estación climatológica Humboldt (Figura 1), que acumuló y registró información cada 30 minutos de las principales variables climáticas del período 1 de enero del 2014 al 30 de abril del 2015, proporcionados por el Instituto Nacional de Meteorología e Hidrología del Ecuador (INAMHI). El procesamiento se lo realizó a nivel horario (mm hora-1), pero para el análisis del comportamiento se los agregó a escala diaria $(\mathrm{mm}$ día ${ }^{-1}$ ).

Las investigaciones en el páramo (Buytaert, Iñiguez, Celleri, De Biévre, Wyseure y Deckers, 2006; Guzmán et al., 2015) determinaron valores de Kc de 0,42 para representan condiciones naturales del ecosistema, con vegetación homogénea y con intervención relativamente baja por agricultura y ganadería; y registros de Ks de 1 que indican contenidos de humedad en el suelo sobre capacidad de campo cercanos a punto de saturación; particularidad encontrada en los páramos de la región.

\subsubsection{Caudal}

Para la medición del caudal se construyó un vertedero de pared delgada de sección combinada triangular y rectangular y se instaló una sonda de nivel automática Instrumentación Northwest (INW) marca PT2X, que acumuló datos cada cinco minutos (Torres, 2016) del período 1 de enero del 2014 al 
30 de abril del 2015. Con la información de las mediciones de altura del nivel de agua, que relaciona la presión de la columna de agua por encima del punto cero del vertedero y la presión atmosférica; y considerando las dimensiones de la infraestructura hidráulica se transformaron los datos a caudal aplicando la relación de Sotelo Avila (1974) para la sección triangular y la de Kindsvater y Carter (1957) para la sección rectangular.

Con la información procesada se separó el caudal base del flujo de tormenta, con fundamento en el algoritmo numérico de los dos parámetros propuesto por Chapman (Chapman, 1999), con una recesión de dos días y elaborado en base a un filtro digital a nivel diario (Ochoa-Tocachi et al., 2016).

\subsubsection{Balance hídrico}

Para el cálculo del balance hídrico anual se utilizó la ecuación general (Ecuación 2) aplicada al ecosistema páramo (Buytaert, Iñiguez, Celleri, De Biévre, Wyseure y Deckers, 2006; Guzmán et al., 2015) y adaptada a las características del sitio.

$$
P=E+E T r+Q+\frac{d s}{d t}+L
$$

Donde $\mathrm{P}$ es la lámina de precipitación en mm, E representa la cantidad de agua interceptada por la vegetación y evaporada directamente desde el dosel en $\mathrm{mm}$, que para las zonas de páramo es relativamente baja (Tobón y Gil Morales, 2007) y según (Buytaert, Célleri, De Bièvre, Cisneros, Wyseure, Deckers y Hofstede, 2006) este término puede ser excluido en la ecuación. ETr es la evapotranspiración real (mm), Q representa el caudal de la cuenca en $\mathrm{mm}$, $\mathrm{L}$ se refiere a la percolación profunda en $\mathrm{mm}$ $\mathrm{y}$ ds/dt que describe el cambio en el contenido de humedad del suelo en mm, y según Torres (2016) para Jatunsacha es igual o cercano a cero, afirmación corroborada por Buytaert, Célleri, De Bièvre, Cisneros, Wyseure, Deckers y Hofstede (2006) en un estudio realizado en una microcuenca de páramo al sur del Ecuador.

\section{Resultados y discusión}

\subsection{Variabilidad temporal de la lluvia}

La precipitación anual para la cuenca de estudio fue de $840,2 \mathrm{~mm}$, siendo octubre el mes más lluvioso con 145,8 mm y febrero el más seco con 17,5 mm. Los resultados en este estudio concuerdan con la información revisada en la bibliografía, refiriéndose a registros de precipitación que varían entre $700 \mathrm{~mm}$ y $3000 \mathrm{~mm}$ para los páramos sudamericanos (Buytaert, Iniguez y De Bievre, 2007; Buytaert, Iñiguez, Celleri, De Biévre, Wyseure y Deckers, 2006; Celleri et al., 2007; Crespo et al., 2011; Tobón y Morales, 2007).

El ciclo anual presentó una distribución bimodal (Figura 2) con cuatro períodos marcados: 1) dos temporadas lluviosas que iniciaron de marzo a mayo y una segunda concentrada en el mes de octubre; 2) dos períodos secos que se presentaron en los meses de diciembre, enero, julio y agosto; 3) la transición de seco a húmedo que comprende los meses de febrero y septiembre y 4) la transición de húmedo a seco que incluye los registros de junio y noviembre.

Varios autores (Villacís, 2008; Vuille, Bradley y Keimig, 2000) explican que el comportamiento de la precipitación a escala intra-anual en la cuenca en estudio está influenciada por masas de aire húmedo provenientes de la cuenca Amazónica, por el movimiento de la Zona de Convergencia Intertropical ZCIT, y por la influencia del Niño-Oscilación del Sur (ENSO) en sus dos fases (El Niño y La Niña). Adicionalmente, (Villacís, 2008) menciona en su investigación que los valores de precipitación de las cuencas localizadas al oeste del Antisana sufren una disminución debido al "efecto pantalla" del volcán, que actúa como una barrera natural para las masas de aire húmedo que se originan en la Amazonía.

\subsection{Caracterización de los eventos de pre- cipitación}

Durante el período de estudio se registraron 819 eventos con cantidades de lluvia que varían de 0,1 $\mathrm{mm}$ a $8,9 \mathrm{~mm}$, duraciones de $5 \mathrm{~min}$ a $452,75 \mathrm{~min}$ e intensidades entre $0,21 \mathrm{~mm} \mathrm{~h}^{-1}$ a $3,6 \mathrm{~mm} \mathrm{~h}^{-1}$. El período lluvioso presentó el mayor número de eventos con 362 y el rango más amplio en la cantidad de lluvia; la transición de seco a húmedo registró el menor número de eventos (112) y rangos con baja longitud en las variables duración e intensidad. En el período seco se registraron 221 eventos con el rango más corto en cantidad de lluvia; por último la transición de húmedo a seco definido por los 124 eventos presenta el rango más alto en duración (Tabla 1). 
Tabla 1. Características de los eventos de precipitación para los períodos seco, lluvioso, transición seco a húmedo y la transición húmedo a seco.

\begin{tabular}{ccccc}
\hline Período & $\begin{array}{c}\text { Frecuencia } \\
\text { de eventos }\end{array}$ & $\begin{array}{c}\text { Cantidad } \\
(\mathbf{m m})^{*}\end{array}$ & $\begin{array}{c}\text { Duración } \\
\mathbf{( m i n )}^{*}\end{array}$ & $\begin{array}{c}\text { Intensidad } \\
\left(\mathbf{m m ~ h} \mathbf{~ h}^{-1} \mathbf{~}^{*}\right.\end{array}$ \\
\hline Seco & 221 & $0,1-6,5$ & $5-332,5$ & $0,2-2,76$ \\
$\begin{array}{c}\text { Lluvioso } \\
\text { Transición }\end{array}$ & 362 & $0,1-10,69$ & $5-499,75$ & $0,22-3,6$ \\
$\begin{array}{c}\text { Seco - Húmedo } \\
\text { Transición }\end{array}$ & 112 & $0,1-8,9$ & $5-323,6$ & $0,32-3,4$ \\
$\begin{array}{c}\text { Húmedo - Seco } \\
\text { Período completo }\end{array}$ & 124 & $0,1-8,3$ & $5-543,5$ & $0,2-4,5$ \\
\hline
\end{tabular}

* Se utilizó el rango conformado por el percentil 2.5 y el percentil 97.5 como límites superior e inferior tomado de la función de distribución de probabilidades de cada variable.

Analizando los períodos propuestos (Tabla 1) no se observa una tendencia estacional de la precipitación; estas afirmaciones concuerdan con lo expresado por (Celleri et al., 2007), refiriéndose a que la lluvia en los páramos al sur del Ecuador no se concentra en la estación húmeda. Por otro lado, los resultados muestran un aumento en el número de eventos y en la variable cantidad para la época lluviosa, y aunque no se puede explicar con certeza el proceso tal vez pueda estar relacionado con la existencia de dos patrones de precipitación, diferentes en el año, que generan la bimodalidad sugerida por la entrada de aire húmedo provenientes de la cuenca Amazónica y de la región costera del pacífico (Villacís, 2008; Vuille, Bradley y Keimig, 2000).

Con base en los resultados y a la información bibliográfica revisada (Buytaert, Célleri, De Bièvre, Cisneros, Wyseure, Deckers y Hofstede, 2006; Celleri et al., 2007; Tobón y Arroyave, 2007) la precipitación de la cuenca investigada se caracteriza por presentar 819 eventos de corta duración (5 minutos $-7,5$ horas $)$, bajo volumen $(0,1 \mathrm{~mm}-8,9 \mathrm{~mm})$ y baja intensidad $\left(0,21 \mathrm{~mm} \mathrm{~h}^{-1}-3,6 \mathrm{~mm} \mathrm{~h}^{-1}\right)$, típico de la distribución de las lluvias en los páramos neotropicales.

\subsection{Variabilidad temporal de la humedad del suelo}

La humedad del suelo en el horizonte A (Figura 3), a escala diaria para el periodo de estudio presenta dos temporadas de máximo contenido de humedad, el 12 de mayo del 2014 y el 11 de noviembre del 2014 con $0,74 \mathrm{~cm}^{3} \mathrm{~cm}^{-3}$ y $0,73 \mathrm{~cm}^{3} \mathrm{~cm}^{-3}$, respectivamente; originando que la humedad esté próximo a punto de saturación $\left(0,75 \mathrm{~cm}^{3} \mathrm{~cm}^{-3}\right)$. Su pico más bajo se registró el 16 de enero del 2015 con un valor de $0,51 \mathrm{~cm}^{3} \mathrm{~cm}^{-3}$, lo que generó que la curva de humedad del suelo disminuya por debajo de la capacidad de campo $\left(0,6 \mathrm{~cm}^{3} \mathrm{~cm}^{-3}\right)$; esta tendencia se presentó en cuatro periodos adicionales que corresponden al 13 de septiembre del 2014, 7 de febrero del 2015, 23 de febrero del 2015 y 13 de marzo del 2015. 


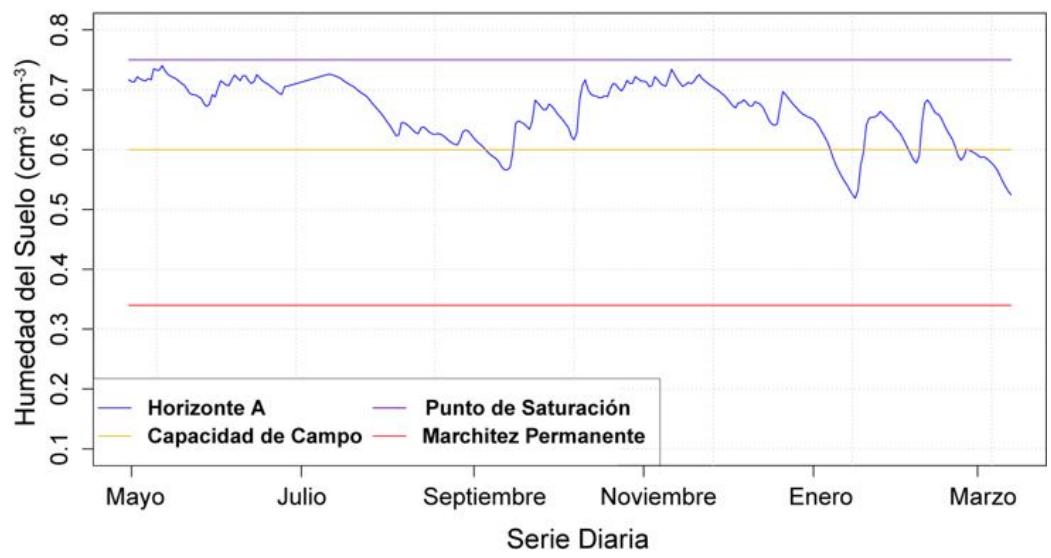

Figura 3. Dinámica diaria de la humedad del suelo en el horizonte superficial A.

La humedad del suelo en el horizonte superficial muestra un comportamiento muy dinámico inclusive mayor que el horizonte $2 \mathrm{Ab}$, debido al impacto de las frecuente lluvias, a la evapotranspiración y al alto contenido de raíces en los primeros centímetros de suelo que generan flujos preferenciales (Blume, Zehe y Bronstert, 2007; Buytaert, Celleri, Willems, De Bievre y Wyseure, 2006; Hofstede, 1995); de los 304 días monitoreados se observó que la mayor parte del periodo, $85 \%$ de datos diarios, los registros estuvieron sobre la capacidad de campo (Figura 3). De igual forma, si se considera la profundidad del horizonte $\mathrm{A}(21,3 \mathrm{~cm})$, su potencial de almacenamiento sería de $108,6 \mathrm{~mm}$ a $156,5 \mathrm{~mm}$. De acuerdo con estos resultados, el horizonte superficial presenta gran capacidad de retención de agua, caracterizado por una fuerte estructura, alta porosidad $(72,4 \%)$, baja densidad aparente $\left(0,64 \mathrm{Mg} \mathrm{m}^{-3}\right)$ y alto contenido de materia orgánica $(11,9 \%)$ típico de los Andisoles (Nanzyo, Shoji y Dahlgren, 1993; Buytaert, Iniguez y De Bievre, 2007).

La dinámica del horizonte $2 \mathrm{Ab}$ es más estable, con valores entre capacidad de campo $\left(0,56 \mathrm{~cm}^{3}\right.$ $\left.\mathrm{cm}^{-3}\right)$ y punto de saturación $\left(0,68 \mathrm{~cm}^{3} \mathrm{~cm}^{-3}\right)$ (Figura 4), con un pico máximo que se originó el 11 de noviembre del $2014 \mathrm{de} 0,65 \mathrm{~cm}^{3} \mathrm{~cm}^{-3}$. El punto más bajo de la curva de humedad del suelo diaria se presentó el 13 de marzo del 2015 con un registro de 0,58 $\mathrm{cm}^{3} \mathrm{~cm}^{-3}$, dato superior al encontrado en el pico más bajo del horizonte superficial $\left(0,51 \mathrm{~cm}^{3} \mathrm{~cm}^{-3}\right)$. El alto contenido de agua en el horizonte $2 \mathrm{Ab}$ se debe principalmente a la baja densidad aparente con $0,70 \mathrm{Mg} \mathrm{m}^{-3}$, alta porosidad de $68,36 \%$ y alto contenido de materia orgánica con 5,8\% (Nanzyo, Shoji y Dahlgren, 1993; Buytaert, Deckers y Wyseure, 2007); que permite una capacidad de almacenamiento entre $365,4 \mathrm{~mm}$ y $326,43 \mathrm{~mm}$, al considerar una profundidad del horizonte $2 \mathrm{Ab}$ de $55,8 \mathrm{~cm}$.

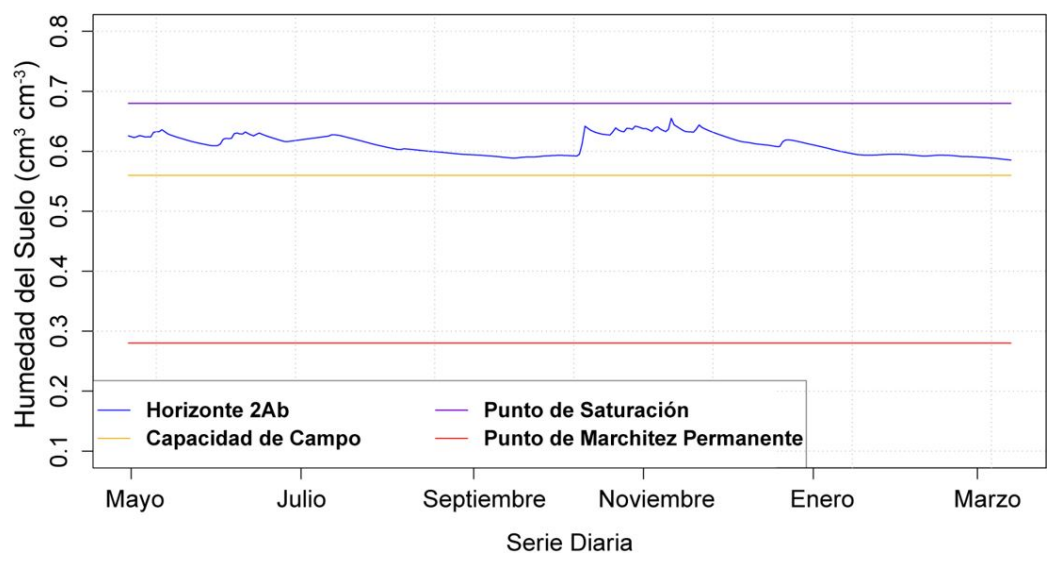

Figura 4. Dinámica diaria de la humedad del suelo en el horizonte $2 \mathrm{Ab}$. 


\subsection{Evapotranspiración real a nivel de cuenca}

La ETr anual calculada para la cuenca Jatunsacha fue de $237,66 \mathrm{~mm}$, con un promedio diario de 0,65 $\mathrm{mm}$ día $^{-1}$ y con registros entre $0,28 \mathrm{~mm}$ día- 1 a 1,04 $\mathrm{mm}$ día $^{-1}$, que corresponden a los limites inferior y superior de la distribución de probabilidades de los datos; su comportamiento fue estacionario para el período de análisis, mostrando valores extremos de 1,68 $\mathrm{mm} \mathrm{día}^{-1}$ en los primeros días de enero del 2014 y mínimos de $0,07 \mathrm{~mm}_{\text {día }}{ }^{-1}$ en junio del 2014 (Figura 5).

Los valores relativamente bajos encontrados en el parámetro evapotranspiración real (Figura 5) para la cuenca en estudio que forma parte de los páramos del Antisana concuerda con los reportes para los páramos de la región con variaciones entre 0,6 mm día ${ }^{-1}$ a 2,2 $\mathrm{mm}$ día ${ }^{-1}$ (?Buytaert, Célleri, De Bièvre, Cisneros, Wyseure, Deckers y Hofstede, 2006; Favier et al., 2008); y su comportamiento depende de las condiciones meteorológicas (Allen et al., 1998), características morfofisiológicas de la vegetación (Cleef, 1981; Hofstede, 1995; Buytaert, Célleri, De Bièvre, Cisneros, Wyseure, Deckers y Hofstede, 2006) y al contenido de agua en el suelo (Figura 3 y Figura 4).

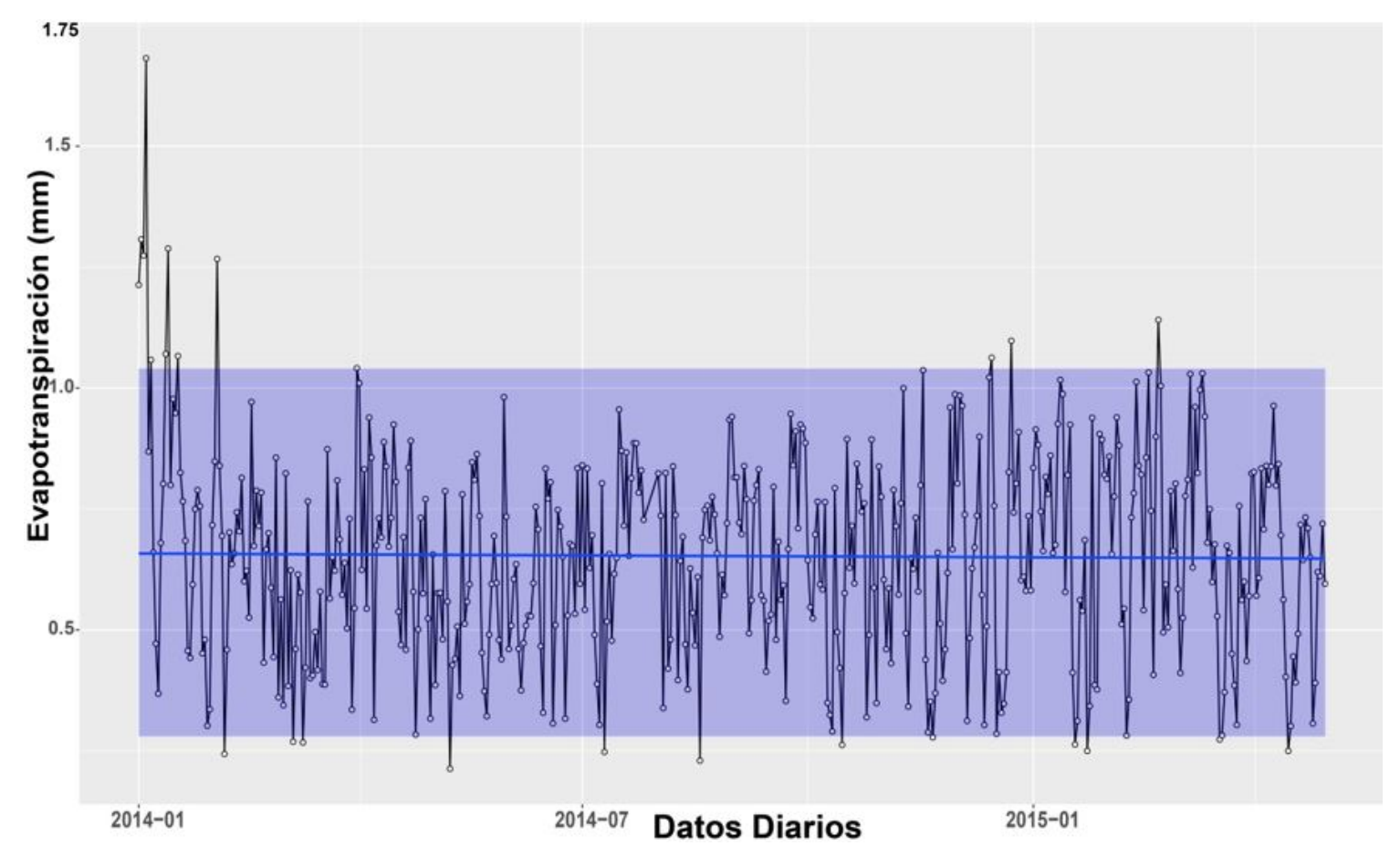

Figura 5. Comportamiento temporal de la evapotranspiración real.

\subsection{Comportamiento temporal del caudal y sus componentes}

Los resultados de la series de tiempo de caudal muestran un valor anual de $89,20 \mathrm{~mm}$, es decir un flujo promedio diario de 5,6 $1 \mathrm{~s}^{-1}$, conformado por dos componentes: los flujos base y escorrentía. La respuesta hidrológica en el páramo de Jatunsacha describe un comportamiento dinámico, con un pico máximo de 88,13 $1 \mathrm{~s}^{-1}$ el 11 de octubre del 2014 y con un registro bajo de $0,39 \mathrm{l} \mathrm{s}^{-1}$ el 21 de febrero del 2016 (Figura 6), que depende de la presencia de lluvias constantes (Tabla 1), alta capacidad de almacenamiento de agua de los suelos con registros $(>80 \%)$ entre capacidad de campo y punto de saturación (Figura 3 y Figura 4), baja evapotranspiración (Figura 5) y alta capacidad de infiltración (Buytaert, 2004; Buytaert, Iñiguez, Celleri, De Biévre, Wyseure y Deckers, 2006; Buytaert, Celleri, Willems, De Bievre y Wyseure, 2006; Sarmiento, 2000). 


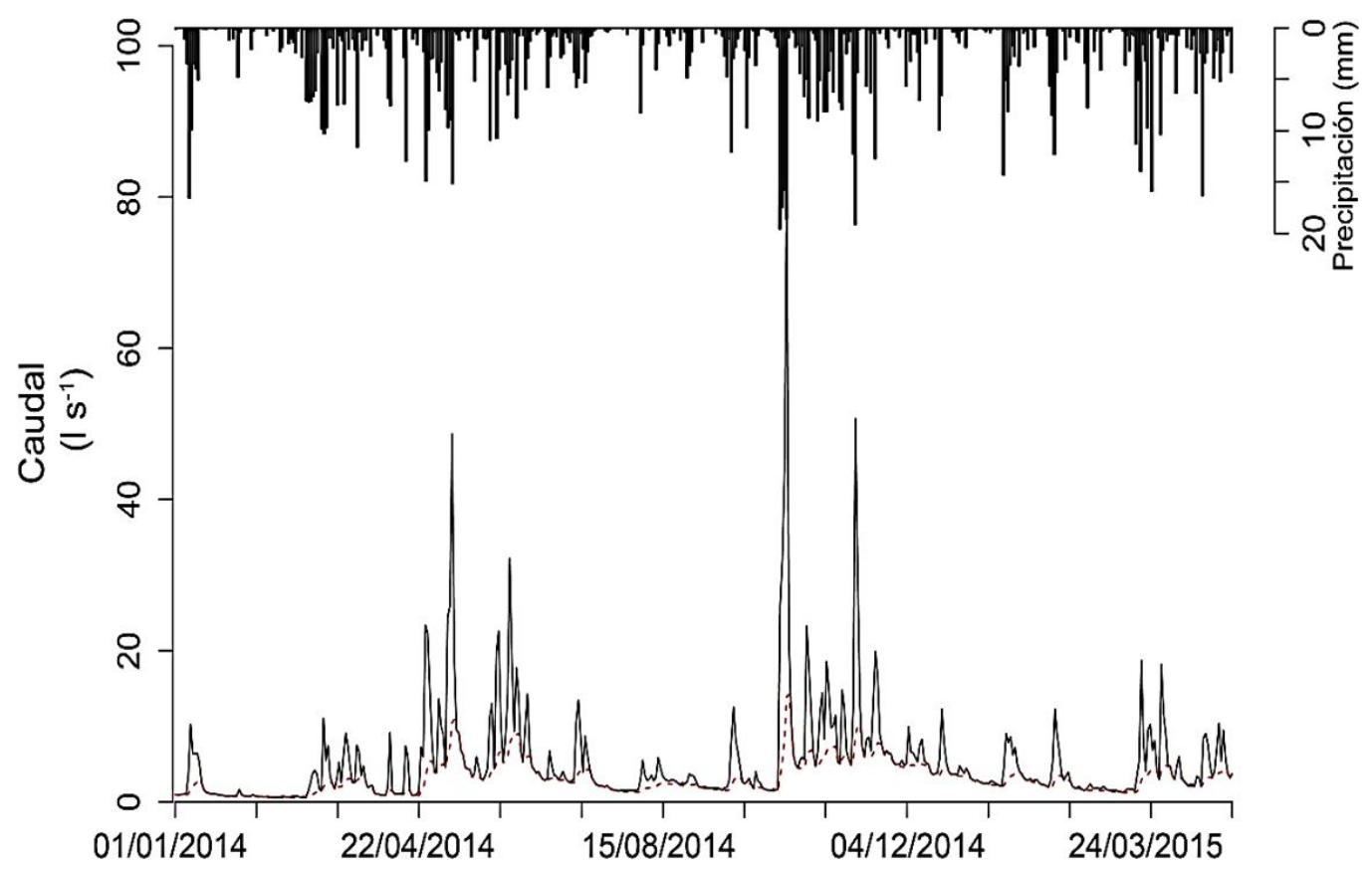

Figura 6. Comportamiento temporal de los caudales base y de tormenta a escala de cuenca con cobertura de páramo.

Los caudales base, que en los ecosistema de montaña representa la capacidad de regulación hídrica, para la cuenca en estudio es relativamente alta $(60 \%)$, con un valor promedio de $3,41 \mathrm{~s} \mathrm{~s}^{-1}$; permitiendo un flujo constante de agua especialmente en períodos de sequía (Hofstede et al., 2003; Buytaert, Célleri, De Bièvre, Cisneros, Wyseure, Deckers y Hofstede, 2006; Buytaert, Celleri, Willems, De Bievre y Wyseure, 2006). Por otro lado, los resultados de las series de tiempo de caudal presentan un registro promedio por escorrentía superficial de 2,2 1 $\mathrm{s}^{-1}$, que implica una contribución del $40 \%$ al flujo total y se genera por flujo superficial de saturación (Chow, 1996) que se produce cuando el contenido de agua subsuperficial satura el suelo desde capas inferiores, contribuyendo de manera signifi- cativa a la precipitación efectiva; afirmación corroborada por investigaciones al sur de Ecuador (Buytaert, Iniguez y De Bievre, 2007; Crespo et al., 2011).

\subsection{Balance hídrico}

El balance hídrico anual en Jatunsacha presenta estimaciones por entrada de precipitación de 840,24 $\mathrm{mm}$ y flujos de salida por evapotranspiración de 237,66 mm (Tabla ??) y por caudal de $89,20 \mathrm{~mm}$, lo que implica un coeficiente de escorrentía del 10,6\%; siendo esta medida el rendimiento hídrico de la cuenca que para Jatunsacha fue relativamente bajo; dato similar al rango encontrado en la investigación de Ochoa-Tocachi et al. (2016) para cuencas de páramo ubicadas en los volcanes Antisana y Pichincha con cifras entre $8 \%$ y $13 \%$.

Tabla 2. Balance hídrico anual en la cuenca Jatunsacha

\begin{tabular}{lcc}
\hline \multicolumn{1}{c}{ Parámetros } & Valores $\mathbf{( m m )}$ & Porcentaje de la precipitación (\%) \\
\hline Precipitación & 840,24 & \\
Caudal & 89,20 & 10,6 \\
Evapotranspiración real & 237,66 & 28,3 \\
Percolación profunda & 513,38 & 61,1 \\
\hline
\end{tabular}


Con la instrumentación de precisión instalada y con información de calidad procesada en cada uno de los parámetros del balance hídrico anual analizados, se cuantificó la pérdida de agua por percolación profunda para recarga de acuíferos, en base a la ecuación general, proyectando un registro de $513,38 \mathrm{~mm}$ que representa el $61,1 \%$ de la precipitación (Tabla ??), siendo el principal parámetro que genera flujo de salida del sistema, y aparentemente esto se genera por la geoestructuras porosa en la zona (Coltorti y Ollier, 2000).

\section{Conclusiones y Recomendaciones}

El comportamiento de los parámetros que integran la dinámica hidrológica en los páramo de la cuenca Jatunsacha están caracterizados por: a) una precipitación con tendencial anual no estacionaria que describen un comportamiento bimodal con eventos de lluvia frecuentes de baja intensidad y volumen; b) por un contenido de humedad en el suelo entre capacidad de campo y punto de saturación, relacionado con el alto contenido de materia orgánica, alta porosidad, y baja densidad aparente; c) por una evapotranspiración relativamente baja con una dinámica muy estacionaria, d) por un caudal constante conformado por un flujo base que aporta un $60 \%$ al caudal y por un flujo de escorrentía que implica una contribución del $40 \%$ al caudal. De igual forma, la zona en estudio presenta un e) coeficiente de escorrentía relativamente bajo y f) una percolación alta debido a la presencia de geoestructuras porosas.

\section{Agradecimientos}

Los autores agradecen la colaboración del Fondo para la Protección del Agua FONAG y del Instituto Nacional de Meteorología e Hidrología del Ecuador INAMHI por la entrega de datos para el estudio.

\section{Referencias}

Allen, R, L Pereira, Raes y M Smith. 1998. Crop Evaporation: guidelines for computing crop water requirements. Roma: FAO - Food and Agriculture Organization of the United Nations.
Alvarado, C. 2009. “Caracterización Hidrogeológica de las Vertientes Occidentales del Volcán Antisana como parte de los Estudios de los Glaciares y Páramos frente al Cambio Climático." Universidad Central del Ecuador, Quito, Ecuador .

Barberi, Franco, Mauro Coltelli, Giorgio Ferrara, Fabrizio Innocenti, José M Navarro y Roberto Santacroce. 1988. "Plio-quaternary volcanism in Ecuador." Geological Magazine 125(1):1-14.

Black, C. A. 1965. Method of Soil Analysis,. Method of Soil Analysis, American Society of Agronomists.

Blume, T, E Zehe y A Bronstert. 2007. "Use of soil moisture dynamics and patterns for the investigation of runoff generation processes with emphasis on preferential flow." Hydrology and Earth System Sciences Discussions 4(4):2587-2624.

Bourdon, Erwan, Jean-philippe Eissen, Michel Monzier, Claude Robin, Hervé Martin, Joseph Cotten y Minard L Hall. 2002. "Adakite-like lavas from Antisana Volcano (Ecuador): evidence for slab melt metasomatism beneath Andean Northern Volcanic Zone." Journal of Petrology 43(2):199-217.

Buytaert, Wouter. 2004. The properties of the soils of the south Ecuadorian paramo and the impact of land use changes on their hydrology $\mathrm{PhD}$ thesis Katholieke Universiteit Leuven.

Buytaert, Wouter, Francisco Cuesta-Camacho y Conrado Tobón. 2011. "Potential impacts of climate change on the environmental services of humid tropical alpine regions." Global Ecology and Biogeography 20(1):19-33.

Buytaert, Wouter, J Sevink, B De Leeuw y Jozef Deckers. 2005. "Clay mineralogy of the soils in the south Ecuadorian páramo region." Geoderma 127(1-2):114-129.

Buytaert, Wouter, Jozef Deckers y Guido Wyseure. 2007. "Regional variability of volcanic ash soils in south Ecuador: The relation with parent material, climate and land use." Catena 70(2):143-154.

Buytaert, Wouter, Rolando Célleri, Bert De Bièvre, Felipe Cisneros, Guido Wyseure, Jozef Deckers y Robert Hofstede. 2006. "Human impact on the hydrology of the Andean páramos." Earth-Science Reviews 79(1-2):53-72. 
Buytaert, Wouter, Rolando Celleri, Patrick Willems, Bert De Bievre y Guido Wyseure. 2006. “Spatial and temporal rainfall variability in mountainous areas: A case study from the south Ecuadorian Andes." Journal of hydrology 329(3-4):413-421.

Buytaert, Wouter, Vicente Iñiguez, Rolando Celleri, Bert De Biévre, Guido Wyseure y J Deckers. 2006. Analysis of the water balance of small paramo catchments in south Ecuador. In Environmental role of wetlands in headwaters. Springer pp. 271-281.

Buytaert, Wouter, Vicente Iniguez y Bert De Bievre. 2007. "The effects of afforestation and cultivation on water yield in the Andean páramo." Forest ecology and management 251(1-2):22-30.

Celleri, Rolando, Patrick Willems, Wouter Buytaert y Jan Feyen. 2007. "Space-time rainfall variability in the Paute basin, Ecuadorian Andes." Hydrological Processes: An International Journal 21(24):33163327.

Célleri, Rolando y Jan Feyen. 2009. "The hydrology of tropical Andean ecosystems: importance, knowledge status, and perspectives." Mountain Research and Development 29(4):350-355.

Chapman, Tom. 1999. "A comparison of algorithms for stream flow recession and baseflow separation." Hydrological Processes 13(5):701-714.

Chow, Ven Te. 1996. Hidrología aplicada. McGrawHill. Online: https://goo.gl/sjGRkB.

Cleef, A.M. 1981. "The Vegetation of the Páramos of the Colombian Cordillera Oriental." Mededelingen van het Botanisch Museum en Herbarium van de Rijksuniversiteit te Utrecht pp. 1-320. Online: https://goo.gl/RbfDF3.

Coltorti, My CD Ollier. 2000. "Geomorphic and tectonic evolution of the Ecuadorian Andes." Geomorphology 32(1-2):1-19.

Crespo, Patricio Javier, Jan Feyen, Wouter Buytaert, Amelie Bücker, Lutz Breuer, Hans-Georg Frede y Marco Ramírez. 2011. “Identifying controls of the rainfall-runoff response of small catchments in the tropical Andes (Ecuador)." Journal of Hydrology 407(1-4):164-174.

Favier, Vincent, Anne Coudrain, Eric Cadier, Bernard Francou, Edgar Ayabaca, Luis Maisincho,
Estelle Praderio, Marcos Villacis y Patrick Wagnon. 2008. "Evidence of groundwater flow on Antizana ice-covered volcano, Ecuador/Mise en évidence d'écoulements souterrains sur le volcan englacé Antizana, Equateur." Hydrological Sciences Journal 53(1):278-291.

Grossman, R., Reinsch. T. 2002. Bulk density and linear extensibility. Methods of soil analysis. Part 4. Book Series No. 5. Soil Science Society of America, Inc. Madison, WI. chapter 2.1, pp. 201-228.

Guarderas, R. 2015. Calibración de los Sensores de Humedad TDR CS625. Technical report FONAG - EPN Quito, Pichincha, Ecuador: .

Guzmán, Pablo, Okke Batelaan, Marijke Huysmans y Guido Wyseure. 2015. "Comparative analysis of baseflow characteristics of two Andean catchments, Ecuador." Hydrological Processes 29(14):3051-3064.

Harden, Carol P., James Hartsig, Kathleen A. Farley, Jaehoon Lee y Leah L. Bremer. 2013. "Effects of Land-Use Change on Water in Andean PÃ $\tilde{j}_{\text {ramo }}$ Grassland Soils." Annals of the Association of American Geographers 103(2):375-384. Online: https: //doi.org/10.1080/00045608.2013.754655.

Hofstede, Robert G. M. 1995. “The effects of grazing and burning on soil and plant nutrient concentrations in Colombian páramo grasslands." Plant and Soil 173(1):111-132. Online: https:/ / doi.org/ 10.1007/BF00155524

Hofstede, Robert, Pool Segarra, Vásconez Mena et al. 2003. The páramos of the world. Global Peatland Initiative/NC-IUCN/EcoCiencia.

Holwerda, F., R. Burkard, W. Eugster, F. N. Scatena, A. G. C. A. Meesters y L. A. Bruijnzeel. 2006. "Estimating fog deposition at a Puerto Rican elfin cloud forest site: comparison of the water budget and eddy covariance methods." Hydrological Processes 20(13):2669-2692. Online: https:/ / doi.org/ 10.1002/hyp.6065.

Jones, Scott B., Jon M. Wraith y Dani Or. 2002. "Time domain reflectometry measurement principles and applications." Hydrological Processes 16(1):141-153. Online: https://goo.gl/qA2tY3.

Kindsvater, Carl E. y Rolland W. Carter. 1957. “Discharge characteristics of rectangular thin-plate 
weirs." Journal of the Hydraulics Division 83(6):136.

Klute, A. 1986a. Methods of Soil Analysis. p. 1187.

Klute, A. y C. Dirksen. 1996. Hydraulic conductivity and diffusivity: Laboratory methods. Methods of Soil Analysis. Part I: Physical and mineralogical properties. 2nd ed. Agronomy, vol. 9, American Society of Agronomy and Soil Science of America, Madison, pp. 687-732.

Klute, Arnold. 1986b. "Water retention: laboratory methods." Methods of soil analysis: part 1 physical and mineralogical methods (methodsofsoilan1):635662.

Lavenu, A., C. Noblet, M.G. Bonhomme, A. Egüez, F. Dugas y G. Vivier. 1992. "New Ki-Ar age dates of Neogene and Quaternary volcanic rocks from the Ecuadorian Andes: Implications for the relationship between sedimentation, volcanism, and tectonics." Journal of South American Earth Sciences 5(3):309-320. Online: https://doi.org/10.1016/ 0895-9811(92)90028-W.

Luteyn, James L, SP Churchill, D Griffin III, SR Gradstein, HJM Sipman y A Gavilanes. 1999. "A checklist of plant diversity, geographical distribution, and botanical literature." New York Bot Gard 84:1-278.

Nanzyo, M., S. Shoji y R. Dahlgren. 1993. Chapter 7 Physical Characteristics of Volcanic Ash Soils. In Volcanic Ash Soils, ed. Sadao Shoji, Masami Nanzyo y Randy Dahlgren. Vol. 21 of Developments in Soil Science Elsevier pp. 189-207. Online: https: / /doi.org/10.1016/S0166-2481(08)70268-X.

Nystuen, Jeffrey A. 1999. "Relative Performance of Automatic Rain Gauges under Different Rainfall Conditions." Journal of Atmospheric and Oceanic Technology 16(8):1025-1043. Online: https://doi.org/10.1175/1520-0426(1999) 016<1025:RPOARG > 2.0.CO;2.

Ochoa-Tocachi, Boris F., Wouter Buytaert, Bert De Bievre, Rolando Célleri, Patricio Crespo, Marcos Villacís, Carlos A. Llerena, Luis Acosta, Mauricio Villazón, Mario Guallpa, Junior Gil-Ríos, Paola Fuentes, Dimas Olaya, Paúl Viñas, Gerver Rojas y Sandro Arias. 2016. "Impacts of land use on the hydrological response of tropical Andean catchments." Hydrological Processes 30(22):40744089. Online: https://goo.gl/8EZ1em.
Sarmiento, L. 2000. "Water Balance and Soil Loss Under Long Fallow Agriculture in the Venezuelan Andes." Mountain Research and Development 20(3):2246-2253. Online: https://goo.gl/vtctem.

Sklenár, Petr y Henrik Balslev. 2005. "Superp"aramo plant species diversity and phytogeography in Ecuador." Flora - Morphology, Distribution, Functional Ecology of Plants 200(5):416433. Online: https://doi.org/10.1016/j.flora. 2004.12.006.

Sotelo Avila, Gilberto. 1974. Hidráulica General: Fundamentos. Vol. 1 Limusa.

Tobón, C y E. G. Gil Morales. 2007. “Capacidad de interceptación de la niebla por la vegetación de los páramos andinos." Avances en Recrusos Hidráulicos 15(35-46).

Tobón, C y F Arroyave. 2007. Inputs by fog and horizontal precipitation to the páramo ecosystems and their contribution to the water balance. In Proceedings Fourth International Conference on Frog Collection and Dew. pp. 233-236.

Tobón, Conrado y Eydith Girleza Gil Morales. 2007. "Capacidad de interceptación de la niebla por la vegetación de los páramos andinos." Avances en recursos Hidráulicos (15).

Tokay, Ali, David B. Wolff, Katherine R. Wolff y Paul Bashor. 2003. "Rain Gauge and Disdrometer Measurements during the Keys Area Microphysics Project (KAMP)." Journal of Atmospheric and Oceanic Technology 20(11):1460-1477. Online: https://doi.org/10.1175/1520-0426(2003) 020<1460:RGADMD>2.0.CO;2 .

Torres, S. 2016. Parámetros de Control de la Dinámica Hidrológica del Páramo de Antisana-Ecuador. Master's thesis Tesis de Maestría de Bosques y Conservación Ambiental. Universidad Nacional de Colombia, sede Medellín.

Villacís, M. 2008. Discipline : Météorologie, océanographie physique et physique de l'environnement $\mathrm{PhD}$ thesis Ecole Doctorale : Systemes Integres en Biologie, Agronomie, Geosciences, Hydrosciences, Environnement. Universite Montpellier II. Online: https://goo.gl/wP4DFb. 
Vuille, Mathias, Raymond S. Bradley y Frank Keimig. 2000. "Climate Variability in the Andes of Ecuador and Its Relation to Tropical Pacific and Atlantic Sea Surface Temperature Anomalies."
Journal of Climate 13(14):2520-2535. Online: https: //doi.org/10.1175/1520-0442(2000)013<2520:

CVITAO $>2.0 . \mathrm{CO} ; 2$. 